\title{
Characterizing the intrinsic correlations of scale-free networks
}

\author{
J. B. de Brito ${ }^{1,2}$, C. I. N. Sampaio Filho ${ }^{1 *}$, A. A. Moreira ${ }^{1}$, J. S. Andrade Jr. ${ }^{1}$ \\ ${ }^{1}$ Departamento de Física, Universidade Federal do Ceará, 60451-970 Fortaleza, Ceará, Brazil \\ ${ }^{2}$ Departamento de Física, Universidade Estadual do Piauí, 64.002-150 Teresina, Piauí, Brazil
}

(Dated: October 2, 2018)

\begin{abstract}
Very often, when studying topological or dynamical properties of random scale-free networks, it is tacitly assumed that degree-degree correlations are not present. However, simple constraints, such as the absence of multiple edges and self-loops, can give rise to intrinsic correlations in these structures. In the same way that Fermionic correlations in thermodynamic systems are relevant only in the limit of low temperature, the intrinsic correlations in scale-free networks are relevant only when the extreme values for the degrees grow faster than the square-root of the network size. In this situation, these correlations can significantly affect the dependence of the average degree of the nearest neighbors of a given vertice on this vertices's degree. Here, we introduce an analytical approach that is capable to predict the functional form of this property. Moreover, our results indicate that random scale-free networks models are not self-averaging, that is, the second moment of their degree distribution may vary orders of magnitude among different realizations. Finally, we argue that the intrinsic correlations investigated here may have profound impact on the critical properties of random scale-free networks.
\end{abstract}

PACS numbers: 89.75.Hc, 64.60.Ak, 89.20.Hh, 89.75.Da

\section{INTRODUCTION}

Very often, the network representation of a real system displays the so called scale-free property [1-4]. These networks typically exhibit a degree distribution in the form of a power law, $P(k) \sim k^{-\gamma}$, with the most connected nodes reaching a degree $k$ orders of magnitude higher than the average degree $\langle k\rangle$. In fact, in most models of scale-free networks the degree of the most connected nodes diverges at the thermodynamic limit, $K_{\max } \sim N^{\theta}$, with $N$ being the number of nodes in the network and $\theta$ some positive exponent. If $\gamma<3$ and with $K_{\max }$ diverging, the second moment, $\left\langle k^{2}\right\rangle$, also diverges, leading to some surprising results [5], such as the critical point of several models in scale-free networks going to zero in the thermodynamic limit [6 12].

The exponent $\theta$ controlling the divergence of $K_{\max }$ can also affect significantly the structure of the network [13]. Simple extreme value statistics [13 15] shows that, even if no other constraint is imposed, the most connected node in the network should have a degree of the order of $K_{\max } \sim N^{\theta_{n}}$, with $\theta_{n}=1 /(\gamma-1)$, which represents the natural cutoff of scale-free networks [13]. However, using the natural cutoff may lead to unexpected properties in the network structure. Typically, if the links are placed at random, one should expect the average number of links between a pair of nodes do be given by $n_{i j}=k_{i} k_{j} / N\langle k\rangle$ [16, 17]. If the nodes $i$ and $j$ are extremely connected nodes, one may expect their degree to be of the order of $K_{\max }$. In this case, for scale-free networks with $\gamma<3$, the maximum value for $n_{i j}$ grows with the network size and should reach values above one,

*Correspondence to: cesar@fisica.ufc.br that is, for these scale-free networks one should expect multiple connections between the most connected nodes. However, it is usually the case that network models do not account for multiple connections, which would lead to the presence of intrinsic correlations [13]. As a matter of fact, these intrinsic correlations have been observed in different random network models [13, 18]. Moreover, since they can be related to correlations of Fermionic systems due to the exclusion principle, a practical analytical treatment becomes possible [17]. More specifically, in analogy with correlations of Fermionic systems that may become irrelevant at sufficiently high temperatures, the intrinsic correlations in networks may practically disappear if a more restrictive constraint is imposed to the extremely connected nodes, $K_{\max } \sim N^{\theta_{s}}$, with $\theta_{s} \leq 1 / 2$. In this case, the maximum expected value for $n_{i j}$ does not diverge with $N$ and multiple connections would not be present even if allowed. This so-called structural cutoff [13] represents a constraint that is strong enough to turn degree-degree correlations in the network negligible.

In this work we investigate the problem of intrinsic correlations in random scale-free networks. This is performed by measuring the dependence of the average connectivity of the nearest neighbors with the degree of the node, $k_{n n}(k)$. We confirm that for scale-free networks the intrinsic correlations lead to a disassortative network, with $k_{n n}$ being depreciated in the most connected nodes. We observe, however, that the particular form for $k_{n n}(k)$ varies widely depending on the network instance, that is, two networks of same size, generated with the same underlying degree distribution, may display highly different dependencies for $k_{n n}(k)$. From our results, we observe that the moments of the degree distribution, $\langle k\rangle$ and $\left\langle k^{2}\right\rangle$, are determinant in the shape of $k_{n n}(k)$. We then use an analytic approach, based on the method of Fermionic correlations [17] to obtain the functional de- 
pendence of $k_{n n}(k)$ given the network size and degree distribution. Since the moments of the degree distribution vary from one realization of the network to another, we need to adjust the minimum and maximum cutoffs of the degree distribution to each realization. Using the properly adjusted degree distribution we observe that our approach is capable of correctly predicting the functional form of $k_{n n}(k)$. Our results show that random scale-free network models are not self-averaging. More precisely, relevant parameters of the network, such as the second moment of the degree distribution, can vary orders of magnitude for different realizations.

\section{THE INTRINSIC CORRELATIONS}

To build our scale-free networks we use the configuration model [19 22. In this model, we start by choosing the degrees of each of node from a power-law distribution, namely, we let the degrees of the nodes to be given by,

$$
k_{i}=\left\lfloor\left[\left(K_{\max }^{-\gamma+1}-K_{\min }^{-\gamma+1}\right) r_{i}+K_{\min }^{-\gamma-1}\right]^{\frac{1}{-\gamma+1}}\right\rfloor,
$$

where $\lfloor\cdot\rfloor$ is the floor function and $r_{i}$ are random numbers uniformly distributed between $0<r_{i}<1$. One should note that, while the degrees $k_{i}$ are integers, the parameters $K_{\max }$ and $K_{\min }$ may assume arbitrary values. After the degrees are determined, the network is constructed by randomly selecting a pair of nodes. The probability of selecting a particular node should be proportional to its remaining degree. Once the pair is selected, a connection should be placed between them and, if the connection is allowed, their remaining degree is decreased by one. Note that we include two important constraints, namely, there is no connection between a node and itself, and there is no redundant connections, that is, each pair of nodes will connect at most once. This later constraint is similar to the exclusion principle of Fermionic particles, being particularly important in scale-free networks with $\gamma<3$ as it leads to the so-called intrinsic correlations. The imposed constraints may result in frustrated attempts to construct the network because at some point, while constructing the network, one may reach a situation in which all the nodes with remaining degree are already connected. In this case, this attempt is rejected and the whole process needs to be restarted by drawing a new set of degrees.

After the network is built, we proceed to calculate the average nearest neighbor degree $k_{n n}(k)$. This is done by computing the average degree of the nearest neighbors of each nodes, and then averaging over nodes that possess the same degree. In Fig. 1 we show the results obtained for two network realizations generated with the same set of parameters, number of nodes $N=10^{5}, \gamma=2.5$, and $K_{\min }=2$. We tune the parameter $K_{\max +}=N-1$, however, since frustrated attempts are discarded, the most connected nodes of the network never reach this limit.

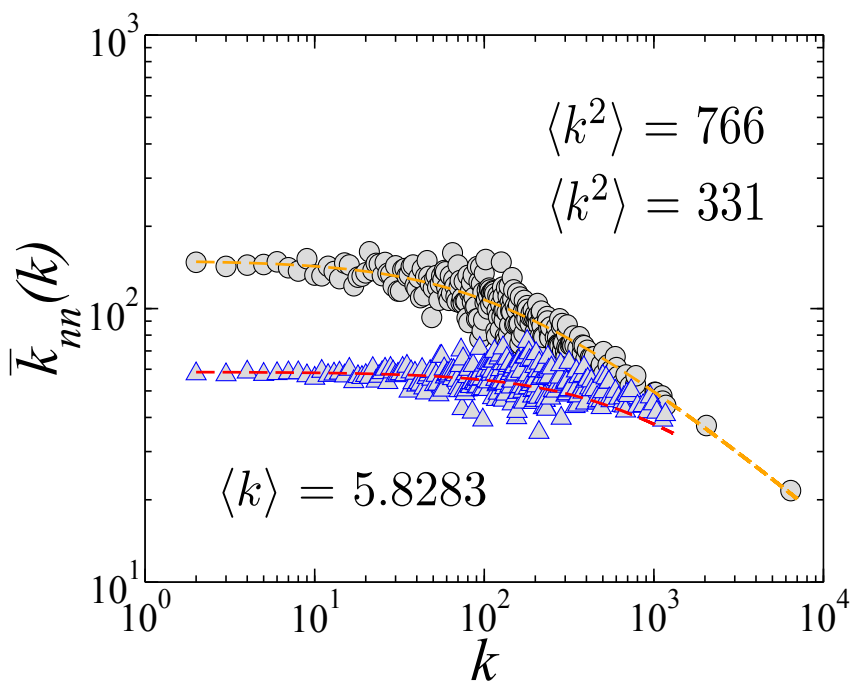

FIG. 1: Average connectivity of the nearest neighbors of vertices with degree $k$, considering two values of $\left\langle k^{2}\right\rangle$ and the same value for the mean degree $\langle k\rangle$. The symbols represent simulations considering the configuration model [19 22]. The dashed lines represent the results of the analytic approach described in the text. Here we considered $N=10^{5}$ for the size of the network and $\gamma=2.5$ for the degree distribution.

Curiously, these two realizations of the model results in quite distinct forms for $k_{n n}(k)$, although both present a clear disassortative trend, with $k_{n n}$ decreasing for the most connected nodes. The difference between these two realizations can be elucidated by noting that, although having similar average degrees, they present very different values for the second moment of the distribution, $\left\langle k^{2}\right\rangle$.

In Fig. 2 we show another two realizations obtained with the same conditions, but now we selected realizations with similar second moments $\left\langle k^{2}\right\rangle$, but distinct averages $\langle k\rangle$. The results for $k_{n n}$ again do not match in this case, although the difference is not as pronounced as in Fig. 1 The fact that the results vary from realization to realization indicate that the network model is not selfaveraging. In this way, to understand the effect of the intrinsic correlation, we need more information than just the network size and the value of the exponent $\gamma$. The absence of the self-averaging property can be observed in Fig. (3), where the probability distributions for the moments $\langle k\rangle$ and $\left\langle k^{2}\right\rangle$ are shown for a set of $10^{6}$ samples of networks built from the configuration model and considering the Fermionic conditions. It is important to notice that the range of the $\left\langle k^{2}\right\rangle$ distribution covers two orders of magnitude.

\section{THE SELF-CONSISTENT EQUATIONS}

By considering that a network is uncorrelated results in an average number of connections between a pair of nodes $i$ and $j$ given by $n_{i j}=k_{i} k_{j} / N\langle k\rangle$. As mentioned 


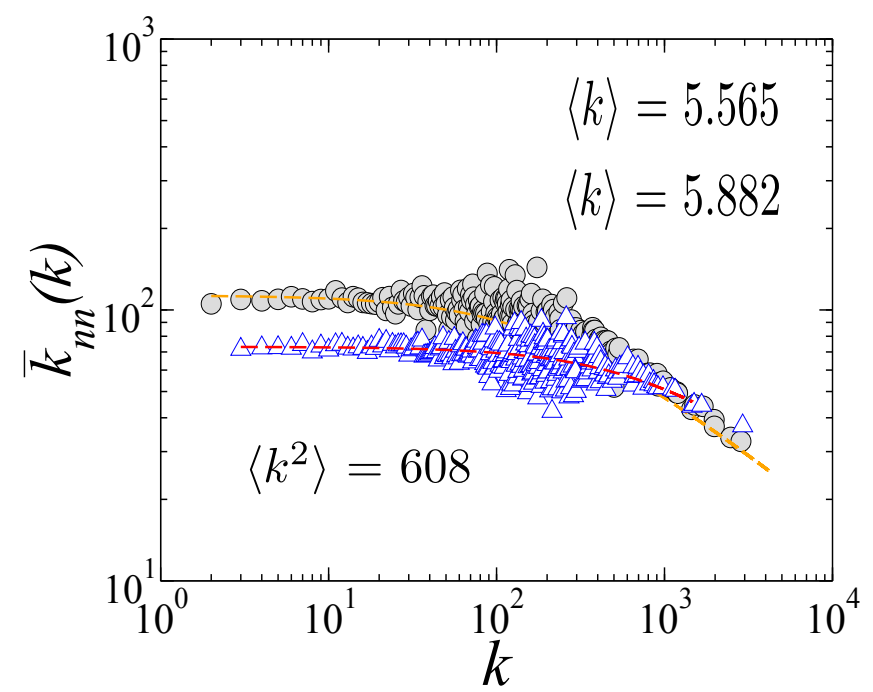

FIG. 2: Average connectivity of the nearest neighbors of vertices with degree $k$, considering the same value for cumulant $\left\langle k^{2}\right\rangle$, and two different values for mean degree $\langle k\rangle$. The symbols represent simulations considering the configuration model [19 22]. The dashed lines represent the results of the analytic approach described in the text. Here we considered $N=10^{5}$ for the size of the network and $\gamma=2.5$ for the degree distribution.
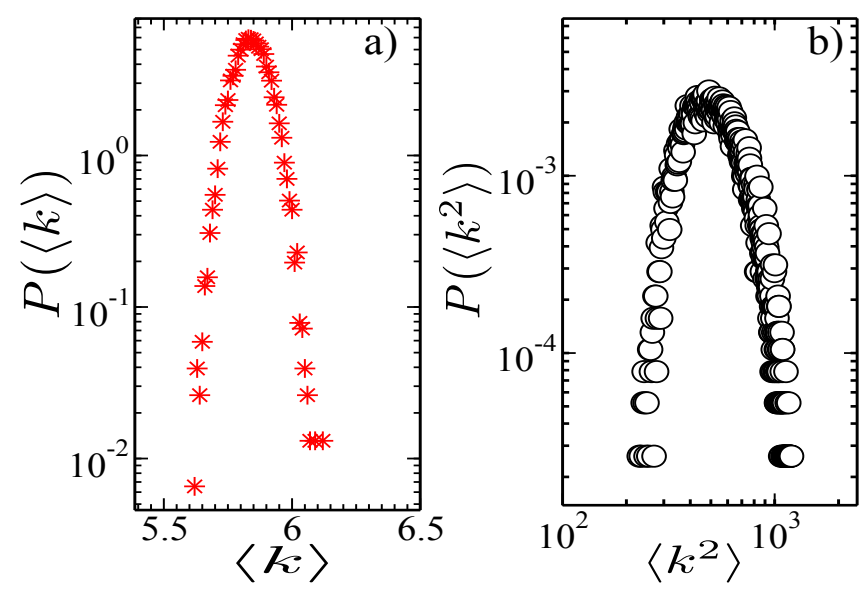

FIG. 3: The probability distributions for the moments $\langle k\rangle$ and $\left\langle k^{2}\right\rangle$. Here we generate $10^{6}$ samples of networks from the configuration model, but do not allow for self and multiple connections.

before, this relation cannot hold for scale-free networks with $\gamma<3$, unless some restriction is imposed to the maximum degree of the nodes, $K_{\max }$. Our goal here is to be able to determine, given a network with distribution $P(k)$ nodes of degree $k$, the dependence on $k$ of the average nearest neighbors $k_{n n}$. The effect of intrinsic correlations on random scale-free networks has been previously studied in [17], where it was shown that the average number of connections between a pair of nodes

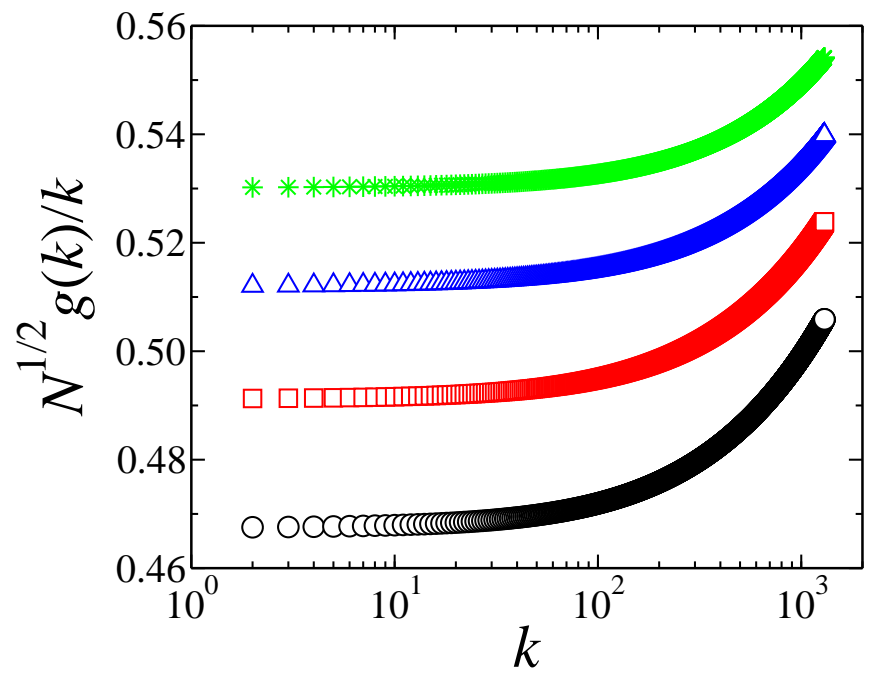

FIG. 4: The $g(k)$ functions obtained from the self-consistent set of equations (3), as calculated for $K_{\min }=2$ and $K_{\max }=$ $N^{1 /(\gamma-1)}$. Here the following cases have been considered: $\gamma=$ 2.5 (circles), $\gamma=2.6$ (rectangles), $\gamma=2.7$ (triangles), and $\gamma=2.8$ (stars).

can be written as,

$$
n_{i j}=\frac{g\left(k_{i}\right) g\left(k_{j}\right)}{1+g\left(k_{i}\right) g\left(k_{j}\right)},
$$

where the form of the function $g(k)$ has to be determined by the consistency of $k_{i}=\sum_{j} n_{i j}$. From that, one obtains the following self-consistent set of equations for the values of $g(k)$ :

$$
g(k)=k\left(\sum_{k^{\prime}} \frac{P(k) g\left(k^{\prime}\right)}{g\left(k^{\prime}\right) g(k)+1}\right)^{-1} .
$$

In order to obtain $g(k)$, we translate Eq. (3) in terms of an iterative process. We start with $g_{0}(k)=k /(N\langle k\rangle)^{1 / 2}$ as an tentative guess for $g(k)$, and place $g_{0}(k)$ on the right side of Eq. (3) to obtain $g_{1}(k)$ on the left side. We then proceed to obtain $g_{2}(k)$ from $g_{1}(k)$ and so on, until we a self-consistent solution is obtained. In Fig. 4 we show the form of $g(k)$ obtained through this process for different expressions of the degree distribution $P(k)$. From $g(k)$, it is possible to obtain the dependence of the average nearest-neighbor degree as,

$$
k_{n n}(k)=\frac{g(k)}{k} \sum_{k^{\prime}} P\left(k^{\prime}\right) \frac{g\left(k^{\prime}\right) k^{\prime}}{g\left(k^{\prime}\right)(g(k)+1)} .
$$

However, as we observed from the results for the configuration model, two important factors need to be taken into account. First, some attempts to build the networks are rejected, therefore the effective degree distribution $P(k)$ may be distorted by this selection criteria. Second, since the model is not self-averaging, the results for a given network may differ greatly from the average result given 
the degree distribution used to generate the network. To obtain $k_{n n}(k)$, we need to determine the dependence we will introduce in the degree distribution $P(k)$ to match each network realization. The simplest approach is to set $P(k)$ exactly as the number of nodes with degree $k$ that we obtained in a given realization. Our simulations show that the obtained form for $k_{n n}(k)$ follows closely the results observed with the configuration model (data not shown).

Alternatively, instead of matching each value of $P(k)$ we tried to reduce the number of parameters by matching only the first and second moments of the degree distribution. To do that we observe that from Eq. (11) we obtain that, on average,

$$
P(k)=N \frac{k^{-\gamma+1}-(k+1)^{-\gamma+1}}{K_{\min }^{-\gamma+1}-K_{\max }^{-\gamma+1}} .
$$

However, considering that $K_{\min }$ can be non-integer, the minimum degree can be written as $k_{\text {min }}=\left\lfloor K_{\text {min }}\right\rfloor$, and

$$
P\left(k_{\min }\right)=N \frac{K_{\min }^{-\gamma+1}-\left(k_{\min }+1\right)^{-\gamma+1}}{K_{\min }^{-\gamma+1}-K_{\max }^{-\gamma+1}}
$$

Therefore, by varying continuously the parameter $K_{\min }$, we reduce continuously the expected number of nodes with the minimum degree $P\left(k_{m i n}\right)$. Now, given the number of nodes $N$ and the exponent $\gamma$, the two free parameters, $K_{\min }$ and $K_{\max }$, can be set to match the values of the first and second moments obtained from a particular random network realization. The results from the configuration model presented in Figs. (11) and (2) are compared with the expected forms for the average nearestneighbor degree $k_{n n}(k)$ obtained with this approach. As depicted, there is good agreement between the distinct approaches.

\section{CONCLUSION}

In this paper we have studied the intrinsic correlations of scale-free networks considering a self-consistent set of equations $g(k)$, in order to analyse the dependence of the average connectivity of nearest neighbors with the degree of nodes. We have confirmed the disassortative behavior of scale-free networks, and demonstrated that the particular form for $k_{n n}(k)$ is strongly dependent on the two first moments of the degree distribution. Moreover, using the properly adjusted degree distributions, based on the minimum and maximum cutoffs of the original degree distribution, we have observed that out approach is capable to provide correct predictions for the functional form of $k_{n n}(k)$. Finally, our results also indicate that random scale-free models are not self-averaging since these models are highly determinant parameters of the network, such as the second moment of the degree distribution, may vary orders of magnitude from one realization to the next. Therefore, it is likely that our results have significant implications on the critical behavior of models defined on complex networks substrates [12, 23 26].

\section{Acknowledgments}

We thank the Brazilian agencies CNPq, CAPES and FUNCAP, the National Institute of Science and Technology for Complex Systems (INCT-SC Brazil.)
[1] R. Albert and A.-L. Barabási, Rev. Mod. Phys. 74, 47 (2002).

[2] G. Caldarelli, A. Capocci, P. De Los Rios, and M. A. Muñoz, Phys. Rev. Lett. 89, 258702 (2002).

[3] S. Boccaletti, V. Latora, Y. Moreno, M. Chavez, and D.-U. Hwang, Physics Reports 424, 175 (2006), ISSN 0370-1573.

[4] A.-L. Barabsi, Science 325, 412 (2009).

[5] A. V. Goltsev, S. N. Dorogovtsev, and J. F. F. Mendes, Phys. Rev. E 67, 026123 (2003).

[6] R. Cohen, K. Erez, D. ben Avraham, and S. Havlin, Phys. Rev. Lett. 85, 4626 (2000).

[7] R. Cohen, K. Erez, D. ben Avraham, and S. Havlin, Phys. Rev. Lett. 86, 3682 (2001).

[8] R. Pastor-Satorras and A. Vespignani, Phys. Rev. Lett. 86, 3200 (2001).

[9] S. N. Dorogovtsev, A. V. Goltsev, and J. F. F. Mendes, Phys. Rev. E 66, 016104 (2002).

[10] R. Pastor-Satorras and A. Vespignani, Phys. Rev. E 65, 035108 (2002).

[11] R. Cohen, S. Havlin, and D. ben Avraham, Phys. Rev. Lett. 91, 247901 (2003).
[12] S. N. Dorogovtsev, A. V. Goltsev, and J. F. F. Mendes, Rev. Mod. Phys. 80, 1275 (2008).

[13] M. Catanzaro, M. Boguñá, and R. Pastor-Satorras, Phys. Rev. E 71, 027103 (2005).

[14] A. A. Moreira, J. S. Andrade, and L. A. Nunes Amaral, Phys. Rev. Lett. 89, 268703 (2002).

[15] M. Ostilli, Phys. Rev. E 89, 022807 (2014).

[16] M. E. J. Newman, Phys. Rev. Lett. 89, 208701 (2002).

[17] J. Park and M. E. J. Newman, Phys. Rev. E 68, 026112 (2003).

[18] J.-S. Lee, K.-I. Goh, B. Kahng, and D. Kim, The European Physical Journal B - Condensed Matter and Complex Systems 49, 231 (2006), ISSN 1434-6028.

[19] A. Bekessy, P. Bekessy, and J. Komlos, Stud. Sci. Math. Hung 7, 343 (1972).

[20] E. A. Bender and E. Canfield, Journal of Combinatorial Theory, Series A 24, 296 (1978), ISSN 0097-3165.

[21] M. Molloy and B. Reed, Random Struct. Algorithms 6, 161 (1995).

[22] M. Molloy and B. Reed, Combinatorics, Probability and Computing 7, 295 (1998), ISSN 1469-2163.

[23] S. Yoon, S. Lee, S.-H. Yook, and Y. Kim, Phys. Rev. E 
75, 046114 (2007).

[24] D.-H. Kim and A. E. Motter, Phys. Rev. Lett. 98, 248701 (2007).

[25] A. V. Goltsev, S. N. Dorogovtsev, and J. F. F. Mendes,
Phys. Rev. E 78, 051105 (2008).

[26] J. P. Gleeson, S. Melnik, and A. Hackett, Phys. Rev. E 81, 066114 (2010). 